\title{
Analysis of a problem in plane geometry discussed in an 11th grade group study session
}

\author{
RICHÁRD RAKAMAZI
}

\begin{abstract}
The main aim of this paper is to show those strategies and proof methods we try to teach in secondary maths education through an interesting geometric problem: Find a relation for the sides of a triangle where an angle is the double of another angle. Is the converse also true? Is it possible to generalize the problem? We try to answer these questions while discussing the upcoming difficulties in detail and presenting more possible solutions. Hopefully the paper can be successfully used in study group sessions and problem solving seminars in secondary schools.
\end{abstract}

Key words and phrases: geometry problem, problem solving, problem solving strategies, different solutions.

ZDM Subject Classification: D50, E50.

Problem 1. Let $A B C \measuredangle=\beta$ and $B C A \measuredangle=2 \beta$ in a triangle $A B C$. Prove that $a b=c^{2}-b^{2}$ !

Solution 1. Rearranging the equation to be proven, we get $c^{2}=b^{2}+a b$, i.e. $c^{2}=b(a+b)$, which is equivalent to $\frac{a+b}{c}=\frac{c}{b}$. This equivalence reminds us of the ratio of the appropriate sides of two similar triangles. Let us look at Figure 1.

Let us measure segment $a$ on the extension of side $A C$ beyond $C$. Triangle $A B C$ is similar to triangle $A B D$, since their corresponding angles are the same. Thus the ratio of the corresponding sides is $\frac{a+b}{c}=\frac{c}{b}$, and this is what we wanted to prove.

Copyright (c) 2013 by University of Debrecen 


$$
\text { "tmcs-rakamazi" — 2013/10/4 — 12:02 — page } 182 \text { — \#2 }
$$

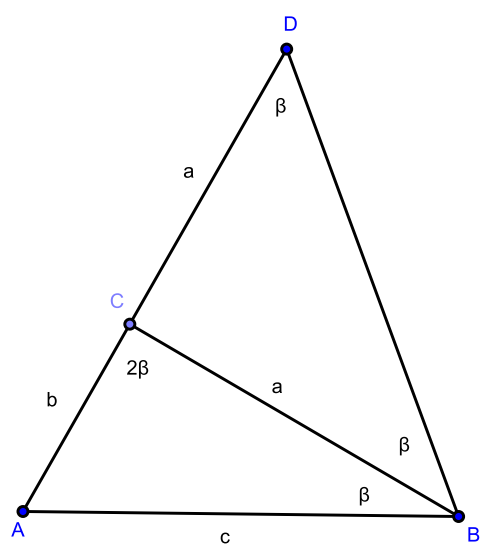

Figure 1

REMARK 2. The presented solution shows an example of analysis i.e. using the backward method [1], [2], [3]. The essence of this is, that we prove $A \Rightarrow B$ by first finding a sufficient condition for $B$ to be a consequence of $B_{1}$. Then we find a sufficient condition $B_{2}$ to imply $B_{1}$, and continue until we reach $B_{n}$. Thus the statement that $A$ implies $B$ follows.

The most important strategic question in this type of thinking is where the statement originates from. For most students this proof process is unusual and even the first step is rather of "ad hoc" nature. If we still want to present this proof in the study group sessions, it is good to discuss the proof of the bisector theorem with the students beforehand! Fortunately the students participating in the session understood and accepted this proof of the statement without difficulty, but among the students' questions the following usually appear: Why do we start the proof exactly like this? How can we come to this idea? In my experience, these are often difficult questions to answer. The majority of the students especially at the study group session experience that in mathematics there are "tricks" having been invented earlier by someone, and applying these we arrive at the desired result. This too is a great advantage of mathematics, not everybody has to figure out everything independently, but we can read and study the works and methods of earlier mathematicians. I usually tell the students, not sidestepping the answer, that it is useful to participate in the study group session also because they can install such technique which later, in a similar problem situation, may be worth "gold". It is usually enough to show this only once to the talented students and 
later they will "think" of the backward method as a possible starting point to solve the problem.

Solution 2. In my opinion the second solution is more straightforward for the students. I have concluded this from the fact, that after giving the problem to the students, more students started their proof this way to arrive at a solution.

Let us use Figure 2.

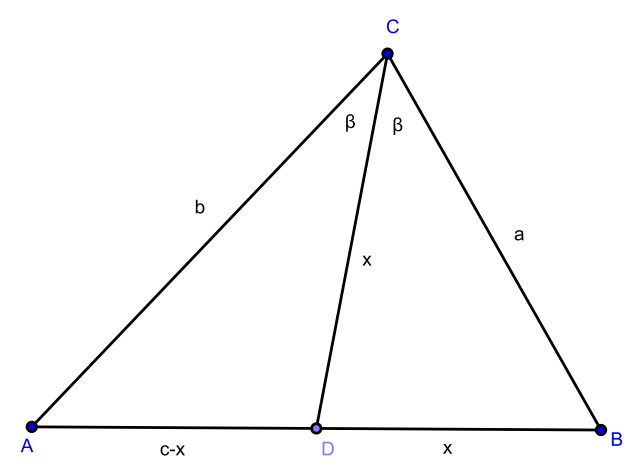

Figure 2

After drawing the bisector of angle $C D$ write the bisector formula for triangle $A B C$.

$$
\frac{x}{c-x}=\frac{a}{b} .
$$

Rearranging the equation we get $x=\frac{a c}{a+b}$. On the other hand, since angle $A D C \measuredangle=2 \beta$, therefore triangle $A D C$ is similar to triangle $A B C$. So, from the equality of the ratios of the corresponding sides we obtain

$$
\frac{b}{c}=\frac{c-x}{b}
$$

from where $x=\frac{c^{2}-b^{2}}{c}$ follows. Comparing the two expressions for $x$ we have:

$$
\begin{aligned}
\frac{a c^{2}}{a+b} & =c^{2}-b^{2} . \\
a c^{2} & =(a+b)\left(c^{2}-b^{2}\right) . \\
a b^{2} & =b\left(c^{2}-b^{2}\right) \Rightarrow a b=c^{2}-b^{2} .
\end{aligned}
$$




$$
\text { "tmcs-rakamazi" — 2013/10/4 — 12:02 — page } 184 \text { — \#4 }
$$

REMARK 3. This proof shows an example of synthesis, which is called the forward method [1]. The key issue for the use of the strategy is, where should we start. This is not always obvious, but in our case the use of the bisector may be a straightforward step, since then an isosceles triangle will appear in the figure. If we show the bisector theorem as an aid before the first solution, then the students will automatically be able to write an equation between the requested quantities. Of course we need to have also another relation which expresses $x$ in terms of $a$, $b, c$. The most talented students immediately recognized the similar triangles and could conclude the proof successfully on their own. But my general experience is that the vast majority of the students had great difficulties with the second step or could not go further at all. It is somewhat a drawback that the geometrical proofs have lately been slightly overshadowed by other topics, and methods using calculation are emphasized instead of synthetic proofs. Therefore, I consider it very important at the group study sessions to include also geometric problems similar to ours which require at least a two-step solution. To improve this type of arguments, in most cases it is very useful to solve problems of type $B$ and $C$ in KöMaL.

Solution 3. Using again the notations of Figure 2 we write cosine formulas for triangles $A D C$ and $B C D$.

$$
\begin{aligned}
x^{2} & =x^{2}+a^{2}-2 a x \cos \beta . \\
(c-x)^{2} & =x^{2}+b^{2}-2 b x \cos \beta .
\end{aligned}
$$

After cancellation the first equation yields (1) $2 x \cos \beta=a$, and using the first equation the second equation implies $(2) c^{2}-b^{2}=2 c x-a b$. Now we write the trigonometric area formula for triangle $A B C$, and obtain

$$
\frac{a b \sin 2 \beta}{2}=\frac{a c \sin \beta}{2} .
$$

We apply the formula for $\sin 2 \beta$ and perform the cancellations to arrive at

$$
2 b \cos \beta=c \Rightarrow 2 b x \cos \beta=c x .
$$

Comparing the last equality with equation (1) we get $c x=a b$, and substituting this into (2) we obtain exactly the relation to be proven.

REMARK 4. This third proof is particularly important for me since I received it from one of my students directly after a study group session. In the 11th 


$$
\text { "tmcs-rakamazi" — 2013/10/4 - 12:02 — page } 185 \text { — \#5 }
$$

Analysis of a problem in plane geometry discussed in an 11th grade group study session $\quad 185$

grade curriculum the sine and cosine laws are discussed, so we can say that he immediately and skilfully applied the fresh knowledge to this problem. After the presentation of the proof we have discussed that the use of trigonometric functions may be a very straightforward idea in such cases, and it is often true that what can be proven by the help of similar triangles is in most cases solvable also via trigonometric functions and vice versa. Most of the students (especially at the study group sessions) who easily deal with the formal calculation steps also feel this kind of solution to be easier, since here we just use the automatic steps of the learnt theorems. Of course it greatly depends on the difficulty level of the assignment, how far the students can get in solving the problem with these mechanical steps. After this I tried to encourage the students to find solutions which use trigonometric functions or other tools not applied earlier.

Solution 4. Dividing both sides of the equation to be proven by the positive $b^{2}$, we obtain $\frac{a}{b}=\left(\frac{c}{b}\right)^{2}-1$. On the basis of the sine law we can easily rewrite the ratio of the sides to the ratio of the sines of the appropriate angles. We use again Figure 1 , and $\sin \left(180^{\circ}-3 \beta\right)=\sin 3 \beta$. Thus it is enough to prove the following equality:

$$
\frac{\sin 3 \beta}{\sin \beta}=\left(\frac{\sin 2 \beta}{\sin \beta}\right)^{2}-1 .
$$

After cancellations we obtain:

$$
\frac{\sin 3 \beta}{\sin \beta}=4 \cos ^{2} \beta-1
$$

By the addition theorem we can obtain $\sin 3 \beta=-4 \sin ^{3} \beta+3 \sin \beta$. Substituting this into the previous equality we have

$$
-4 \sin ^{2} \beta+3=4 \cos ^{2} \beta-1,
$$

from where we can get the obviously correct equality $\sin ^{2} \beta+\cos ^{2} \beta=1$. Since we performed equivalent transformations in each step, therefore the statement is proven.

REMARK 5. The solution mentioned here is mainly interesting from a didactical point of view, since without the last comment it gives an example to a so called incomplete analysis. The students liked to use this proof strategy, but we had to call to their attention separately that if we start from the statement to be proven it always has to be checked whether the steps are reversible since a true statement can originate also from a false statement. When I found this 
solution, the students (except from some hard working, motivated students) have not yet met the addition theorem, so I can apply this proof strategy only a little bit later, but because of the above comment I definitely feel it worth to deal with because of the difficulties arising here. There is a good collection of assignments which require similar ideas and a similar method in the geometry problem book [5] pages 225-226.

Solutions 5-6. The following proofs do not have direct didactical purposes, I only mention them because they are interesting, and I wanted to discuss the problem in greater depth through different solutions. The interesting part of the following solution is that the statement is deduced only from the comparison of certain areas. This method has many applications in mathematics and can be useful in solving a lot of problem situations.

We use the notations of Figure 2. So let us write the area of triangle $A B C$ in two different ways. From the equality of these, we obtain:

$$
\frac{b x \sin \beta}{2}+\frac{a x \sin \beta}{2}=\frac{a c \sin \beta}{2},
$$

from where we get $x=\frac{a c}{a+b}$. On the other hand, we have

$$
\frac{(c-x) x \sin 2 \beta}{2}+\frac{x^{2} \sin \left(180^{\circ}-2 \beta\right)}{2}=\frac{a b \sin 2 \beta}{2} .
$$

After applying the possible cancellations, we get $x=\frac{a b}{c}$.

Comparing the two values obtained for $x$, we arrive at:

$$
\frac{a b}{c}=\frac{a c}{a+b} \Rightarrow a^{2} b+a b^{2}=a c^{2} \Rightarrow a b=c^{2}-b^{2} .
$$

Also the next solution is inventive, where we use the relation $x=\frac{a c}{a+b}$ established previously. Consider Figure 3!

In the right triangle $D E C$ we have $\cos \beta=\frac{a}{2 x}$, from where $x=\frac{a}{2 \cos \beta}$.

Comparing the two equations we get $2 \cos \beta=\frac{a+b}{c}$. Let us write the cosine law for triangle $A B C: b^{2}=a^{2}+c^{2}-2 a c \cos \beta$, and substitute here the value of $\cos \beta$ obtained from the previous formula:

$$
b^{2}-c^{2}=a^{2}-a(a+b) \Rightarrow b^{2}-c^{2}=-a b,
$$

which is equivalent to the statement to be proven.

During secondary school teaching, when we prove a theorem, the question, whether the converse of the theorem is true, arises quite naturally. I too posed this question to the students, and we got the following result: 


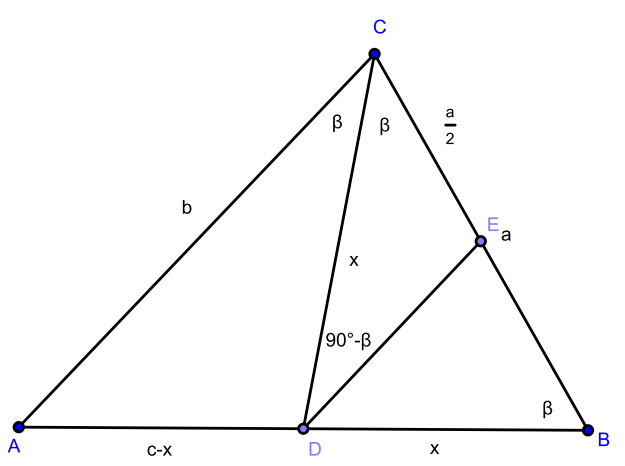

Figure 3

Statement 6. If $a b=c^{2}-b^{2}$ is true in a triangle $A B C$, then $\gamma=2 \beta$.

Solution 1. Let us look at Figure 4!

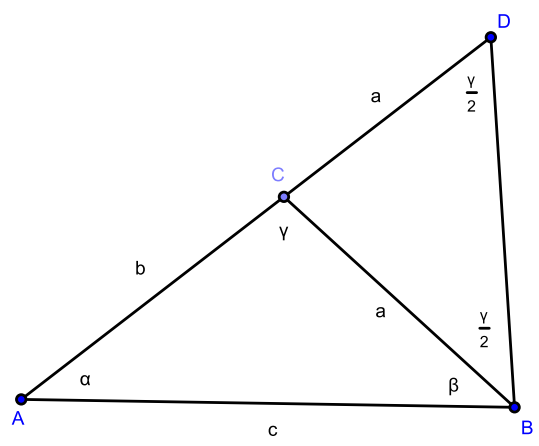

Figure 4

Rearranging the equation $c^{2}-b^{2}=a b$, we get $\frac{c}{b}=\frac{a+b}{c}$. By a basic similarity case of triangles we have $A B C \triangle \sim A D B \triangle$. If two triangles are similar, the corresponding angles are equal. Therefore $\frac{\gamma}{2}=\beta$ is true, from where we have $\gamma=2 \beta$ as stated. 


$$
\text { "tmcs-rakamazi" — 2013/10/4 — 12:02 — page } 188 \text { — \#8 }
$$

Solution 2. Dividing the assumption by $b^{2}$, we arrive at $\left(\frac{c}{b}\right)^{2}-1=\frac{a}{b}$. Using the sine law this relation can be rewritten in form $\sin ^{2} \gamma-\sin ^{2} \beta=\sin \alpha \cdot \sin \beta$. Factoring the left hand side of the equation, and then writing the formulas for $\sin x-\sin y$ and $\sin x+\sin y$, we get

$$
2 \cos \frac{\gamma+\beta}{2} \cdot \sin \frac{\gamma-\beta}{2} \cdot 2 \sin \frac{\gamma+\beta}{2} \cdot \cos \frac{\gamma-\beta}{2}=\sin \alpha \cdot \sin \beta .
$$

By repeated use of the addition formulas, the left hand side of the equation is $\sin (\gamma-\beta) \cdot \sin (\gamma+\beta)$. Since $\gamma+\beta=180^{\circ}-\alpha$ and $\sin \left(180^{\circ}-\alpha\right)=\sin \alpha$, the equation $\sin (\gamma-\beta) \cdot \sin (\gamma+\beta)=\sin \alpha \cdot \sin \beta$ can be reduced to $\sin (\gamma-\beta)=\sin \beta$. The latter however will only be fulfilled for the inner angles of a triangle if $\gamma-\beta=\beta$, hence $\gamma=2 \beta$, and we are done.

Problem 7. What can be stated about the sides of a triangle with $\gamma=n \beta$, where $n>1$ is an integer and $(n+1) \beta<180^{\circ}$ ?

Solution. We examine this general question first in the special case $n=3$. Consider the notations of Figure 5!

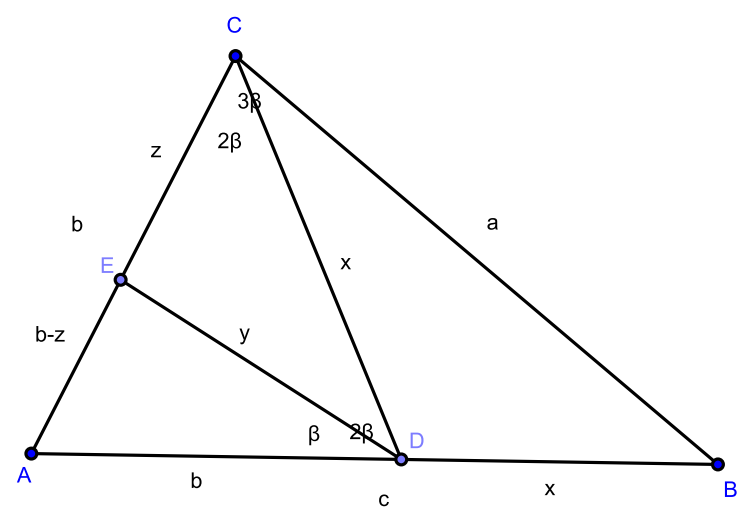

Figure 5

We can apply the relation proven for $n=2$ to the sides of triangle $D C E$, since angle $C$ of the triangle is the double of the angle at $D$. From this we get 


$$
\text { "tmcs-rakamazi" — 2013/10/4 — 12:02 — page } 189 \text { — \#9 }
$$

\begin{tabular}{ll} 
Analysis of a problem in plane geometry discussed in an 11th grade group study session $\quad 189$ \\
\hline
\end{tabular}

$y^{2}-z^{2}=x z$. Furthermore, it is clear that triangle $A D E$ is similar to $A B C$, so the ratios of the corresponding sides are equal.

$$
\frac{b}{c}=\frac{b-z}{b}=\frac{y}{a} \text {. }
$$

Let us apply the bisector theorem to triangle $A D C$ from which we obtain $\frac{b-z}{z}=\frac{b}{x}$. We can express the values $x, y, z$ with the sides of the triangle form this latter equation: $x=\frac{b z}{b-z}=c-b, y=\frac{a b}{c}, z=\frac{b c-b^{2}}{c}$, and substituting these into the first relation we get

$$
\frac{a^{2} b^{2}}{c^{2}}-\frac{\left(b c-b^{2}\right)^{2}}{c^{2}}=(c-b) \cdot \frac{b c-b^{2}}{c} .
$$

After cancellations we obtain

$$
c^{2}-b^{2}=\frac{a^{2} b}{c-b}
$$

Also the converse of the statement is true:

Statement 8. If $c^{2}-b^{2}=\frac{a^{2} b}{c-b}$ is true in a triangle $A B C$, then $\gamma=3 \beta$.

PROOF. Rearranging the equation and dividing both sides by $b^{3}$ we get

$$
\left(\frac{c}{b}-1\right)^{2}\left(\frac{c}{b}+1\right)=\left(\frac{a}{b}\right)^{2}
$$

On the basis of the sine law this implies

$$
\left(\frac{\sin \gamma-\sin \beta}{\sin \beta}\right)^{2} \cdot \frac{\sin \beta+\sin \gamma}{\sin \beta}=\frac{\sin ^{2} \alpha}{\sin ^{2} \beta} .
$$

Applying $\sin \alpha=\sin \left[180^{\circ}-(\beta+\gamma)\right]=\sin (\beta+\gamma)$ and the formulae for $\sin x-\sin y$ and $\sin x+\sin y$, after cancellations we arrive at

$$
\sin (\gamma-\beta) \cdot(\sin \gamma-\sin \beta)=\sin (\gamma+\beta) \cdot \sin \beta
$$

Expanding the sines we obtain

$$
(\sin \gamma \cos \beta-\cos \gamma \sin \beta)(\sin \gamma-\sin \beta)=(\sin \gamma \cos \beta+\cos \gamma \sin \beta) \cdot \sin \beta .
$$

Performing the multiplications and cancellations we get

$$
\sin \gamma \cos \beta-\cos \gamma \sin \beta=2 \cdot \sin \beta \cos \beta .
$$

The latter is equivalent to the equation $\sin (\gamma-\beta)=\sin 2 \beta$, which implies $\gamma=3 \beta$, taking into consideration that $\beta$ and $\gamma$ are the angles of a triangle. 


$$
\text { "tmcs-rakamazi" — 2013/10/4 — 12:02 — page } 190 \text { — \#10 }
$$

We have completely clarified the cases $n=2$ and $n=3$. For the general case let us write two cosine rules for triangle $A B C$. From the equations $b^{2}=$ $a^{2}+c^{2}-2 a c \cos \beta$ and $c^{2}=a^{2}+b^{2}-2 a b \cos n \beta$, we have

$$
\cos \beta=\frac{a^{2}+c^{2}-b^{2}}{2 a c}, \quad \cos n \beta=\frac{a^{2}+b^{2}-c^{2}}{2 a b} .
$$

It is known from algebra that $\cos n \beta$ can be expressed as a polynomial of $\cos \beta$, and this can lead to a relation (getting more and more complex with the increase of $n$ ) between the sides for an arbitrary $n$. Following this procedure for $n=3$ we experience, that it is much more complicated to reach the final form with this method, than it was by the one we obtained by means of a clever trick and some calculations. We are going to carry out the examination for $n=4$ here below. First we need a lemma.

Lemma 9. If $f_{c}$ is the bisector belonging to side $c$ in a triangle $A B C$, then

$$
f_{c}^{2}=a b\left[1-\frac{c^{2}}{(a+b)^{2}}\right]
$$

Proof. Consider Figure 6!

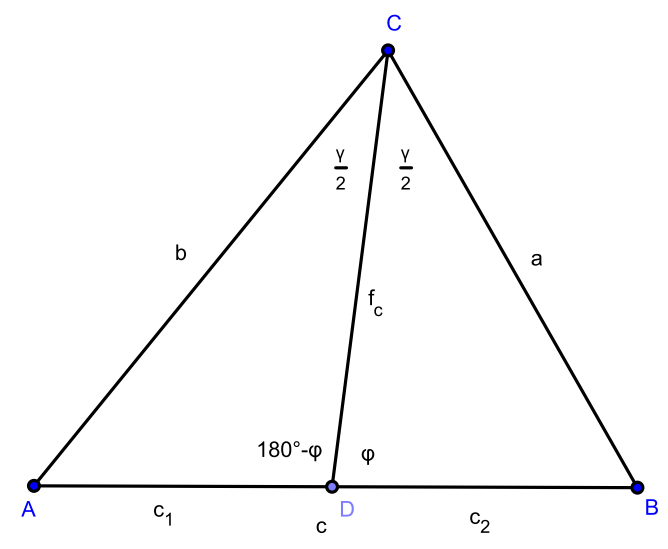

Figure 6

Let us write two cosine formulas for triangles $D B C$ and $A D C$. We have $a^{2}=f_{c}^{2}+c_{2}^{2}-2 f_{c} c_{2} \cos \varphi$ and $b^{2}=f_{c}^{2}+c_{1}^{2}+2 f_{c} c_{1} \cos \varphi$. For the second equation we have used that $\cos \left(180^{\circ}-\varphi\right)=-\cos \varphi$. 


$$
\text { "tmcs-rakamazi" — 2013/10/4 — 12:02 — page } 191 \text { — \#11 }
$$

Analysis of a problem in plane geometry discussed in an 11th grade group study session $\quad 191$

Let us express $\cos \varphi$ from both equations:

$$
\frac{f_{c}^{2}+c_{2}^{2}-a^{2}}{2 f_{c} c_{2}}=\frac{f_{c}^{2}+c_{1}^{2}-b^{2}}{-2 f_{c} c_{1}}
$$

then after cross multiplication and applying the possible cancellations we get equality $a^{2} c_{1}+b^{2} c_{2}=c f_{c}^{2}+c c_{1} c_{2}$. Applying the bisector theorem to triangle $A B C$, we get $\frac{c_{1}}{c_{2}}=\frac{b}{a}$. Since $c_{1}+c_{2}=c$, from this we obtain $c_{1}=\frac{b c}{a+b}$ and $c_{2}=\frac{a c}{a+b}$. If we substitute these terms into the previous relation, we arrive at the statement of the lemma after a short calculation.

We note that there is a very elegant proof of this statement in [4], pages 145-146.

Now we can prove the following:

Problem 10. If $A B C \measuredangle=\beta$ and $B C A \measuredangle=4 \beta$ in a triangle $A B C$, then

$$
c^{2}-b^{2}=\frac{a^{2} b(a+b)}{c^{2}-a b-b^{2}} .
$$

Solution 1. We use Figure 7.



Figure 7

Applying the relation obtained for $n=2$ for triangle $D B C$, we have

$$
(c-x)^{2}-f_{c}^{2}=a f_{c}
$$


Again we use the previously established relations for triangle $A B C$, and obtain $\frac{c-x}{x}=\frac{a}{b}$ and $f_{c}^{2}=a b\left[1-\frac{c^{2}}{(a+b)^{2}}\right]$. After eliminating $x$ from the previous equations, we obtain

$$
\frac{a^{2} c^{2}}{(a+b)^{2}}-a b\left(1-\frac{c^{2}}{(a+b)^{2}}\right)=a \cdot \sqrt{a b} \cdot \sqrt{1-\frac{c^{2}}{(a+b)^{2}}} .
$$

A radical free equivalent form is the following:

$$
\left[a c^{2}-b\left[(a+b)^{2}-c^{2}\right]\right]^{2}-a b\left[(a+b)^{4}-c^{2}(a+b)^{2}\right]=0 .
$$

After cancellation we see that $(a+b)^{2}$ is a factor of the left hand side:

$$
-(a+b)^{2}\left(a^{3} b+a^{2} b^{2}+a b c^{2}-a b^{3}+2 c^{2} b^{2}-b^{4}-c^{4}\right)=0 .
$$

Here the first factor cannot be 0 , so

$$
a^{3} b+a^{2} b^{2}+a b c^{2}-a b^{3}+2 c^{2} b^{2}-b^{4}-c^{4}=0 .
$$

In cases $n=2$ and $n=3$ we saw, that $c^{2}-b^{2}$ can be expressed from the relation. Now we try to do the same, and therefore write our equation in the form $\left(c^{2}-b^{2}\right)^{2}-a b\left(c^{2}-b^{2}\right)=a^{2} b(a+b)$, and express $c^{2}-b^{2}$ :

$$
c^{2}-b^{2}=\frac{a^{2} b(a+b)}{c^{2}-a b-b^{2}} .
$$

Solution 2. We use the addition theorem to get $\cos 4 \beta=8 \cos ^{4} \beta-8 \cos ^{2} \beta+$ 1. On the basis of the previously seen relations

$$
\cos \beta=\frac{a^{2}+c^{2}-b^{2}}{2 a c}, \quad \cos n \beta=\frac{a^{2}+b^{2}-c^{2}}{2 a b}
$$

we arrive at the following equation:

$$
\frac{a^{2}+b^{2}-c^{2}}{2 a b}=8\left(\frac{a^{2}+c^{2}-b^{2}}{2 a c}\right)^{4}-8\left(\frac{a^{2}+c^{2}-b^{2}}{2 a c}\right)^{2}+1
$$

We use the Maple program for the execution of cancellations. The equation is equivalent to the following, very complicated expression:

$$
\begin{gathered}
-(1 / 2) \cdot\left(c^{6} a^{3}-4 b^{7} c^{2}+6 b^{5} c^{4}-4 c^{6} b^{3}+b^{9}-4 b^{3} a^{4} c^{2}-4 b^{3} a^{2} c^{4}+8 b^{5} a^{2} c^{2}\right. \\
\left.-4 b^{7} a^{2}+6 b^{5} a^{4}-4 a^{6} b^{3}+a^{8} b+c^{8} b-a^{5} c^{4}-b^{2} a^{3} c^{4}\right) /\left(a^{4} b c^{4}\right)=0 .
\end{gathered}
$$




$$
\text { "tmcs-rakamazi" — 2013/10/4 — 12:02 — page } 193 \text { — \#13 }
$$

Analysis of a problem in plane geometry discussed in an 11th grade group study session $\quad 193$

Factoring and simplyfying the numerator we get

$$
\begin{aligned}
(c+a-b)(-c+a-b) & \left(b c^{2}+a^{3}+b a^{2}-b^{2} a-b^{3}\right) \\
& \times\left(-b^{4}-b^{3} a+2 c^{2} b^{2}+a^{2} b^{2}+b c^{2} a+b a^{3}-c^{4}\right)=0 .
\end{aligned}
$$

It can be seen that the last factor is exactly the same as the one appearing in the previous solution. None of the first two factors can be 0 due to the triangle inequality. So it is enough to show that under the condition $\gamma=4 \beta$ the third factor cannot be 0 either.

Assume indirectly, that $b c^{2}+a^{3}+b a^{2}-b^{2} a-b^{3}=0$. Rewrite the equation as $a^{3}-b^{3}+a b(a-b)+b c^{2}=0$.

It is clear, that if $a \geq b$, then our statement is true, because the left hand side is definitely positive. If $a<b$, then $\alpha<\beta$, therefore $180^{\circ}-5 \beta<\beta$, thus $\beta>30^{\circ}$. But in this case $\cos 4 \beta<-\frac{1}{2}$, hence $\frac{a^{2}+b^{2}-c^{2}}{2 a b}<-\frac{1}{2}$, from which we get the inequality $a^{2}+b^{2}+a b<c^{2}$. Substituting this into the initial equation we obtain

$$
a^{3}-b^{3}+a b(a-b)+b\left(a^{2}+b^{2}+a b\right)=a^{3}+a^{2} b+b a^{2}<0
$$

which is a contradiction.

\section{References}

[1] A. Ambrus, Introduction to mathematics-didactics, University notes, ELTE Eötvös Kiadó, Budapest, 1995, 92-95 (in Hungarian).

[2] G. Pólya, Mathematical Discovery Volume I: On understanding, learning, and teaching problem solving, John Wiley and Sons Inc., New York, (1962), 6-9.

[3] G. Pólya, How to solve it, A new aspect of mathematical method, Princeton University Press, 1945, 225-232.

[4] J. Strohmajer, Geometric Problem Book I., University notes, Nemzeti Tankönyvkiadó, Budapest, 1977, 145-146 (in Hungarian).

[5] E. Czapáry, Matematics III., Collection of geometric problems, Nemzeti Tankönyvkiadó, Budapest, 2005, 225-226 (in Hungarian).

RICHÁRD RAKAMAZI

JÁNOS NEUMANN SECONDARY SCHOOL

RÁKÓCZI STR. 48.

3300 EGER

HUNGARY

E-mail: raka82@gmail.com

(Received November, 2012) 